\title{
Mechanical Circulatory Support for Peripartum Cardiomyopathy
}

\author{
Tariq Yousuf ${ }^{a}$, e, Jeffrey Ziffra ${ }^{b}$, Jason Kramer ${ }^{c}$, Hesam Keshmiri ${ }^{b}$, Gabriel Sayer ${ }^{d}$
}

\begin{abstract}
Peripartum cardiomyopathy (PPCM) is a rare cause of heart failure that occurs late in pregnancy or during the early postpartum period. Left ventricular assist devices (LVADs) are a form of mechanical circulatory support that are most often implanted for the management of advanced heart failure. We describe a case of PPCM that we managed through implantation of an LVAD with the hope of allowing for myocardial recovery. A 38-year-old female presented 10 days postpartum with chest pain, shortness of breath and hypotension. An echocardiogram revealed a left ventricular ejection fraction (LVEF) of $25 \%$. The patient received an LVAD. Her hemodynamics and symptoms improved dramatically following implantation, and her postoperative course was uncomplicated.
\end{abstract}

Keywords: Cardiac; Cardiomyopathy; Peripartum; Heart failure; Treatment

\section{Introduction}

Peripartum cardiomyopathy (PPCM) is a rare cause of heart failure that occurs late in pregnancy or during the early peripartum period. PPCM has a high risk of mortality with mortality rates reported between $1.36 \%$ and $30 \%$ despite optimal medical therapy $[1,2]$. Recovery rates were reported as $44 \%$ for a partial recovery while $21 \%$ had complete recovery [3].

Left ventricular assist devices (LVADs) are a form of mechanical circulatory support that are most often implanted for the management of advanced heart failure as a bridge to car-

Manuscript accepted for publication April 10, 2015

aAdvocate Christ Medical Center, 105 Covington Ct, Oak Brook, IL 60523, USA

bAdvocate Christ Medical Center Internal Medicine, 4440 W 95th St. Oak Lawn, IL 60451, USA

'Rosalind Franklin Medical School, Chicago, IL, USA

dUniversity of Chicago Division of Cardiology, Chicago, IL, USA

eCorresponding Author: Tariq Yousuf, Advocate Christ Medical Center, 105

Covington Ct, Oak Brook, IL 60523, USA. Email: tmyousuf614@gmail.com

doi: http://dx.doi.org/10.14740/jmc2110w diac transplantation or as destination therapy. In select cases, an LVAD may also be implanted with the hope that it will allow time for the recovery of myocardial function. If sufficient recovery occurs, explantation of the LVAD may be possible [4]. We describe a case of PPCM that we managed through implantation of an LVAD with the hope of allowing for myocardial recovery.

\section{Case Report}

A 38-year-old female with a history of hypertension presented 10 days postpartum with chest pain, shortness of breath and hypotension. The patient was G4P4 and she delivered her fourth child without complication. Several days after her delivery, the patient began to experience symptoms such as chest tightness, shortness of breath and inability to lie flat.

An echocardiogram was performed which revealed a left ventricular ejection fraction (LVEF) of $25 \%$, a left ventricular size of $6.3 \mathrm{~cm}$ and moderate mitral regurgitation. A chest X-ray revealed bilateral pleural effusions and mild pulmonary vascular congestion. Labs were significant for a B-type natriuretic peptide of 3411. Right heart catheterization revealed a mixed venous oxygen saturation of $35 \%$. She was initially managed with an intra-aortic balloon pump (IABP) and milrinone for cardiac support.

She was transferred to our center for consideration of mechanical circulatory support given her severely abnormal hemodynamics. She was maintained on temporary support with the IABP with the hope that she would recover without the need for more durable mechanical assistance. However, her cardiac output remained depressed and repeat echocardiogram showed no sign of improvement. Seven days after transfer to Christ Medical Center, the patient received a HeartMate II LVAD (Thoratec, Inc., Pleasanton, CA). Her hemodynamics and symptoms improved dramatically following implantation, and her postoperative course was uncomplicated.

She has had one readmission since implant due to ventricular tachycardia, which was thought to be secondary to volume depletion. She was successfully cardioverted and has not had any recurrence. She has been maintained on aggressive medical therapy with carvedilol, lisinopril and aldactone. She continues to do well 3 months following LVAD implantation, and her most recent echocardiogram shows an LVEF of 53\% 
with an LV size of $5.0 \mathrm{~cm}$. She is still being monitored to determine if her cardiac recovery is durable and will allow for LVAD explantation.

\section{Discussion}

PPCM is a condition characterized by new and rapid onset heart failure occurring between the last trimester of pregnancy and up to 6 months postpartum. This prevalence of PPCM is between 1:3,000 and 1:15,000 deliveries. Risk factors for PPCM include increased age, multiparity, preeclampsia, the use of tocolytics, twin pregnancy, malnutrition and cocaine abuse. The most common presentations are rapid onset cough, dyspnea and leg edema. PPCM appears similar to dilated cardiomyopathy although it presents acutely or subacutely [5]. There is an increased risk for thrombus and arrhythmias.

Despite optimal medial therapy, PPCM carries a high risk of mortality with reports as high as $30 \%$ [1]. In a case report, $44 \%$ had a partial recovery while $21 \%$ had complete recovery of their ejection fraction [3]. Diagnosis is often clinical but is often made late and is widely misdiagnosed. Treatment includes the same medical therapy given to other forms of heart failure: angiotensin-converting enzyme inhibitors, aldosterone antagonists, diuretics and beta-blockers [5]. PPCM patients who present in cardiogenic shock, which in a recent study was stated at an incidence of $13.1 \%$ of patients, require consideration of more aggressive therapies [6].

An LVAD is a device used in patients with severe heart failure to pump blood from the left ventricle to the ascending aorta [7]. In some cases patients can recover back enough ventricular function to have the mechanical assist device removed. Less than $10 \%$ of patients were reported to have enough recovery to remove mechanical support. This is more commonly seen in dilated cardiomyopathy. The mechanism is related to multiple factors such as unloading, neurohormonal inhibition and the causation of myocardial injury [8]. Another case report has been published involving a postpartum cardiomyopathy requiring LVAD support in which the LVAD was explanted 9 months after implantation and complete recovery of ventricular function was demonstrated 3 years later [9].

In our case, we opted for the use of an LVAD for the management of PPCM due to the persistence of cardiogenic shock despite medical therapy. Our patient has subsequently demonstrated significant recovery of her LV function, and may be a good candidate for LVAD explantation. This demonstrates the importance of early consideration of mechanical circulatory support in severe cases of PPCM that do not rapidly respond to medical therapy.

\section{Learning objective}

PPCM is a rare condition that is characterized by acute onset heart failure. It can resolve with medical therapy and time but there is often an underlying decrease in systolic function. From our case report, the patient did not have underlying cardiac disease and an LVAD was placed to provide circulatory support. These interventions temporarily alleviated the symptoms and further use of the LVAD or even transplantation will be determined after the patient is given time to recover cardiac function.

\section{Conflict of Interest}

No author involved in this project has any conflict of interest to disclose.

\section{References}

1. Hilfiker-Kleiner D, Meyer GP, Schieffer E, Goldmann B, Podewski E, Struman I, Fischer P, et al. Recovery from postpartum cardiomyopathy in 2 patients by blocking prolactin release with bromocriptine. J Am Coll Cardiol. 2007;50(24):2354-2355.

2. Bhattacharyya A, Basra SS, Sen P, Kar B. Peripartum cardiomyopathy: a review. Tex Heart Inst J. 2012;39(1):816.

3. Elkayam U, Tummala PP, Rao K, Akhter MW, Karaalp IS, Wani OR, Hameed A, et al. Maternal and fetal outcomes of subsequent pregnancies in women with peripartum cardiomyopathy. N Engl J Med. 2001;344(21):15671571.

4. Slaughter MS, Rogers JG, Milano CA, Russell SD, Conte JV, Feldman D, Sun B, et al. Advanced heart failure treated with continuous-flow left ventricular assist device. $\mathrm{N}$ Engl J Med. 2009;361(23):2241-2251.

5. Hilfiker-Kleiner D, Schieffer E, Meyer GP, Podewski E, Drexler H. Postpartum cardiomyopathy: a cardiac emergency for gynecologists, general practitioners, internists, pulmonologists, and cardiologists. Dtsch Arztebl Int. 2008;105(44):751-756.

6. Shah I, Shahzeb HM, Shah ST, Faheem M, Rafiullah. P. Cardiomyopathy: risk factors, hospital course and prognosis; experiences at Lady reading Hospital Peshawar. Global Advanced Research Journal of Pharmacy and Pharmacology (GARJPP). 2012;1001-1011.

7. Lietz K, Long JW, Kfoury AG, Slaughter MS, Silver MA, Milano CA, Rogers JG, et al. Outcomes of left ventricular assist device implantation as destination therapy in the post-REMATCH era: implications for patient selection. Circulation. 2007;116(5):497-505.

8. Stevenson LW, Rose EA. Left ventricular assist devices: bridges to transplantation, recovery, and destination for whom? Circulation. 2003;108(25):3059-3063.

9. Oosterom L, de Jonge N, Kirkels J, Klopping C, Lahpor J. Left ventricular assist device as a bridge to recovery in a young woman admitted with peripartum cardiomyopathy. Neth Heart J. 2008;16(12):426-428. 